Thorax (1957), 12, 203.

\title{
THE CONGENITAL DIAPHRAGMATIC HERNIA OF BOCHDALEK
}

\author{
BY \\ MILROY PAUL AND R. KANAGASUNTHERAM \\ From the University of Ceylon
}

(RECEIVED FOR PUBLICATION MARCH 12, 1957)

In 1848 Bochdalek described his dissection of a newborn infant who had died from a congenital hernia through a hiatus in the left postero-lateral part of the diaphragm. Nearly all the abdominal viscera had herniated through the hiatus in the diaphragm into the left pleural cavity. Although Bochdalek stated that he would be making further investigations on this anomaly of development, he published no more papers on this subject. Hernias also occur, though with less frequency, through a hiatus in the right postero-lateral part of the diaphragm. These hernias are also designated hernias of Bochdalek on account of the hiatus on the right side being probably due to the same causes as the hiatus on the left side.

The hernias of Bochdalek are recognizable at clinical examinations, and the diagnosis can be confirmed by radiological examination. The reduction of the displaced viscera from the pleural to the abdominal cavity, with closure of the hiatus in the diaphragm by suture of its edges, is no longer a surgical adventure. The remarkable series of cases recorded by Dickson (1933), Harrington (1945), and Gross (1953) have established that patients with a hernia of Bochdalek can be cured of the hernia by a surgical operation. The operative mortality is comparatively low, being $13 \%$ in the series recorded by Gross.

The success of these operations has focused attention on the early recognition of this condition and on the details of the surgical management of these cases. The records of these cases do not, however, give sufficient details of the arrangement of the viscera which have herniated into the pleural cavity to permit of a reconstruction of the mechanics of the hernia. The concept put forward in this paper that the herniated viscera have a particular arrangement in the pleural cavity has not been suggested as yet. Even the descriptions of the anomaly by Bochdalek (1848), Gruber (1927), and Wells (1954) do not entertain this concept.

\section{Materials AND Methods}

During the course of an investigation into fatal head injuries in the newborn, a full-term male foetus, dying shortly after birth, was found to have most of the abdominal viscera in the left pleural cavity. A full-term female foetus with a similar disposition of the viscera had previously been sent to the Department of Anatomy. The dissection of these two infants with left-sided hernias of Bochdalek was undertaken, as this would give the opportunity for a more detailed investigation of the features of these hernias than would be possible at operations for their repair.

\section{OBSERVATIONS}

General InSPECTION.-The two infants showed no external evidence of any defect and were of normal proportions. On opening the thoracic and abdominal cavities, it was found that the left pleural cavity in both cases was filled to its apex with intestines. The stomach had herniated into the thorax and lay immediately above the left cupola of the diaphragm. The abdominal cavity contained only the liver and a length of large bowel descending in a straight line from the orifice in the diaphragm down to the anus (Fig. 1). The heart and the pericardium were displaced so much towards the right side that the right lung became visible only after rotating the heart to the left. This lung was of normal size and had three lobes.

The left lung was a bilobed rudiment about the size of a walnut lying below the arch of the aorta, and was only seen when the abdominal viscera were lifted out of the pleural cavity.

Abdominal Viscera in left Pleural Cavity. - The oesophagus after entering the abdominal cavity through the oesophageal hiatus of the diaphragm turned sharply and re-entered the left pleural cavity through the hernial orifice in the diaphragm (Fig. 2). It opened into the stomach at this place. The entire stomach lay above the 


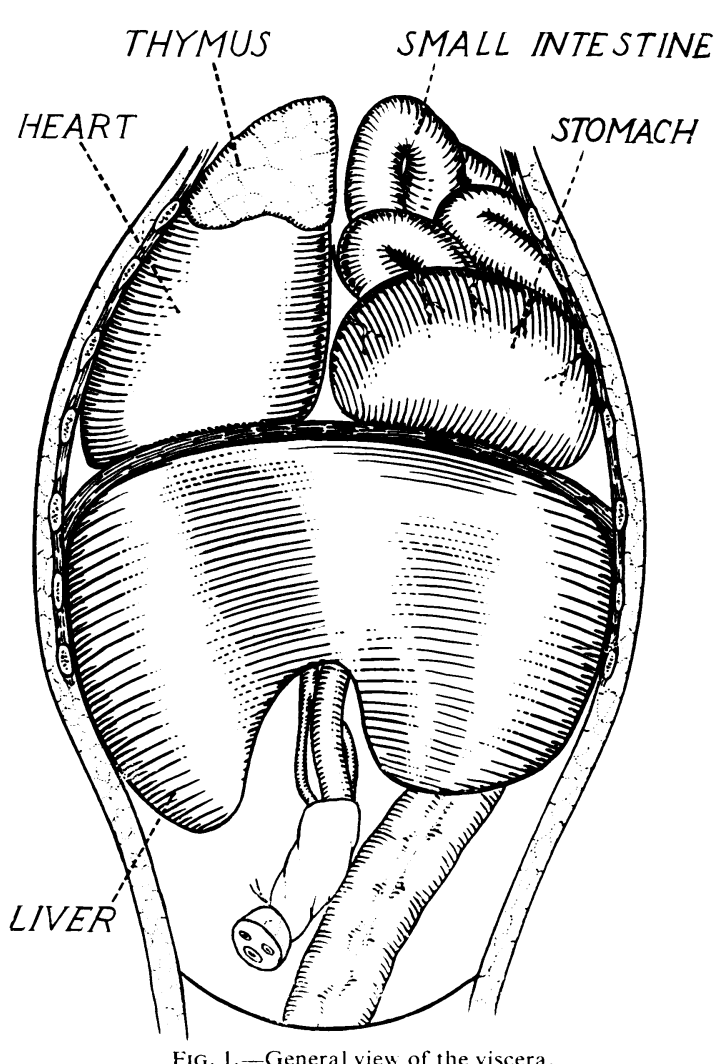

diaphragm with the greater curve directed cranially and with the fundus abutting on the mediastinum. The stomach narrowed towards the pylorus, and the duodenum passed down into the abdominal cavity through the hernial orifice in the diaphragm. The duodenum then made a $\mathrm{U}$ loop in the abdominal cavity and the intestine returned to the left pleural cavity through the same hernial orifice in the diaphragm.

Lying against the greater curve of the stomach with the great omentum attached to it was the transverse colon (Fig. 3). At the fundus of the stomach, the transverse colon bent on itself at a splenic flexure and the spleen lay apposed to the stomach at this place. From the splenic flexure, the colon passed down posterior to the stomach and the ascending limb of the intestine to enter the abdominal cavity through the hernial orifice in the diaphragm. At the pyloric end of the greater curve of the stomach, the transverse colon bent on itself and passed cranially as the ascending colon and caecum. The ileum entered the caecum at this place in the normal manner (Fig. 3).

From the $C$ curve of the duodenum, the intestine ascended into the left pleural cavity and passed behind the stomach and the transverse colon. It continued as the coils of the jejunum and the ileum which were disposed above the transverse colon, filling the left pleural cavity to its apex. The pancreas within the $U$ loop of the duodenum continued upwards into the left pleural cavity through the hernial orifice in the diaphragm up to the spleen (Fig. 2).

Contents of the Abdominal Cavity.-The liver lay across the whole of the upper part of the abdominal cavity, filling it almost entirely (Fig. 1). When the liver was rotated upwards to view its $\vec{N}$ under surface, the gall bladder was exposed and $\omega$ the $U$ loop of duodenum was seen to lie vertically. The hilum of the liver was connected to the $U$ loop of the duodenum by a pedicle in which were the common bile duct, the portal vein, and the hepatis artery (Fig. 2).

Blood Vessels Supplying the Viscera.-The coeliac axis and the superior mesenteric artery had their origin from the abdominal aorta. The left gastric and splenic branches of the coeliac axis

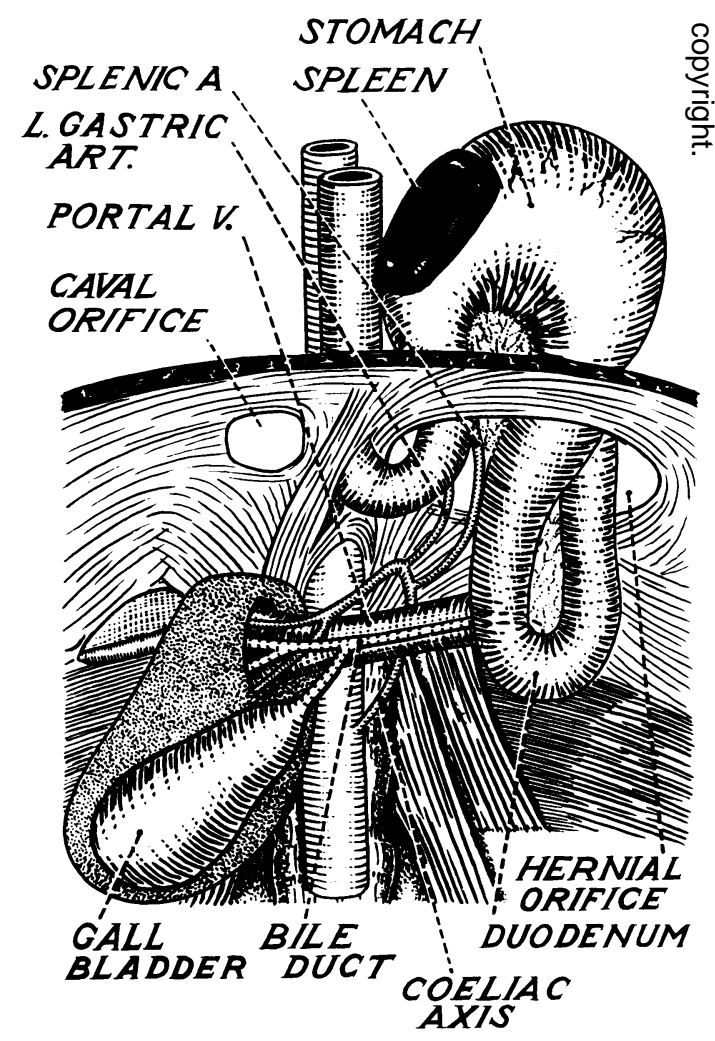

FIG. 2.-The viscera passing through the hernial hiatus with their vascular pedicles. 
and the superior mesenteric artery ascended into the thorax through the diaphragmatic hernial orifice while the hepatic artery passed transversely across from the coeliac axis to the hilum of the liver. In the free border of the lesser omentum were the portal vein, the hepatic artery, and the bile duct, in their normal relationship to each other (Fig. 2).

The Diaphragm.-The hernial orifice was a transversely disposed oval, 3 in. $\times 2$ in., on the left cupola of the diaphragm near its dorsal attachment. This orifice lay entirely within the costal part of the diaphragm. In the male infant, the oval was a $U$ loop between the diaphragmatic fibres arising from the left eleventh rib and those arising from the twelfth rib (Fig. 3). In the female infant, the oval was between muscle fibres attached to the eleventh and twelfth ribs (Fig. 4). At the margins of the orifice the parietal layers of the pleural and peritoneal membranes became continuous with each other.

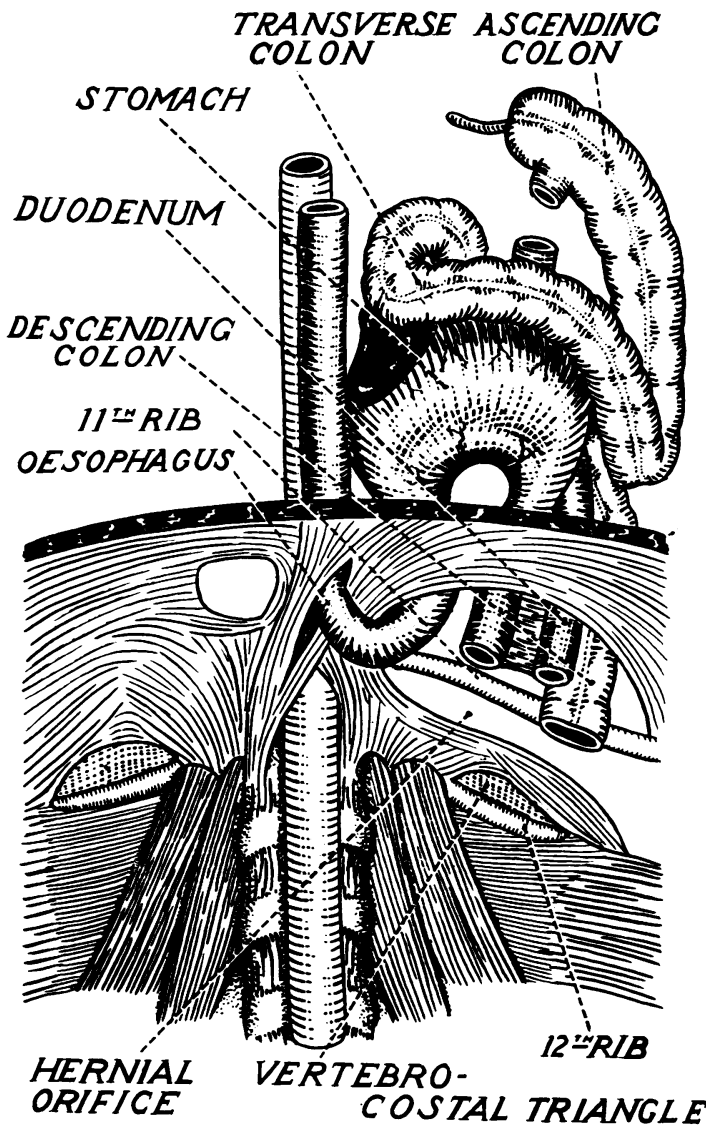

FIG. 3.-The diaphragm in the male body.

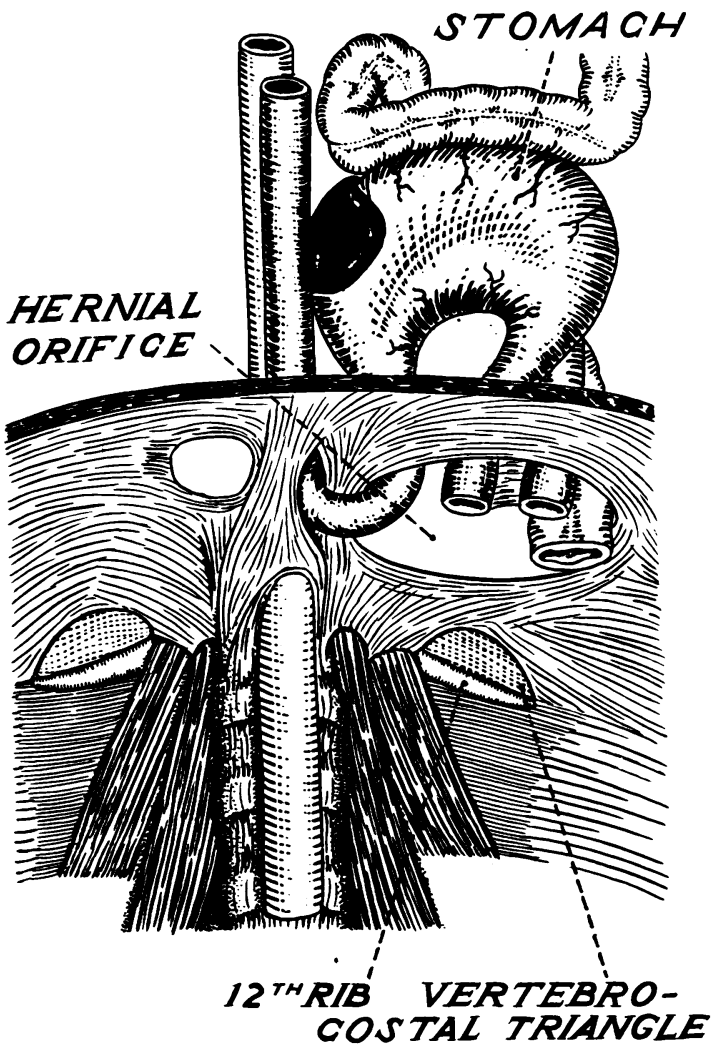

FIG. 4.-The diaphragm in the female body.

The vertebro-costal triangle was posterior to the diaphragmatic hernial orifice in both cases, and in each instance was filled with areolar tissue (Figs. 3 and 4). The medial angle of the hernial orifice was close to the oesophageal hiatus in the diaphragm. The fibres of the right crus spread out like a fan, after forming the oesophageal hiatus, to merge with the rest of the muscle fibres forming the medial angle of the orifice. The left crus was not well developed in either specimen but was present.

\section{Disposition of the Viscera and the Causes OF THEIR HERNIATION}

The identical dispositions of the viscera in these two infants with left-sided hernias of Bochdalek would be explicable only if the viscera had herniated in an orderly sequence. The sequence could have been determined by the relative mobilities of the viscera, if the more mobile viscera were displaced before the less mobile. A survey of the published records of left-sided hernias of Bochdalek is also evidence that the viscera herniate in an orderly sequence, because those viscera which 
herniate are either the small intestines alone, or the small and the large intestines, or the small and large intestines with the stomach (Rickham, 1955).

It is remarkable that writers have recorded these findings without drawing the inference that the viscera must have herniated in the sequence small intestine first, then large intestine, and finally the stomach. This sequence operated despite the fundus of the stomach being at the mouth of the hiatus in the diaphragm, and no case has been recorded in which the stomach has herniated alone through a left-sided hiatus of Bochdalek. The two bodies which were dissected showed that not only do the viscera herniate in an orderly sequence, but that each viscus follows a set path and takes up a set position in the left pleural cavity.

FACTORS RESPONSIBLE FOR HERNiation OF VISCERA.-What determines the herniations of the viscera from the abdominal to the pleural cavity ? The dissections of the two infants suggest that the viscera had been drawn into the left pleural cavity by a pressure gradient. In them, the abdominal cavity had been emptied of every viscus which could move into the left pleural cavity, and the left pleural cavity was so filled that the mediastinum had been displaced well over to the right. This displacement of the mediastinum was all the more remarkable in that both infants had survived birth, and the displacement of the mediastinum had withstood aeration of the right lung while the left lung still remained a rudimentary bud.

What could have determined the pressure gradient which forced the viscera to leave the abdominal cavity? The only sizable viscus remaining in the abdominal cavity was the liver. Could not the liver by its growth have increased the intra-abdominal pressure to such a degree as to cause the herniation of the other viscera into the pleural cavity, especially when there was no sizable viscus here to oppose the invasion, the left lung being a rudimentary bud?

Our observations suggest that the growth of the liver was not solely responsible for determining the pressure gradient, because the abdomen was of normal size and accommodated all the herniated viscera when these were reduced from the pleural cavity. Moreover, the rudimentary size of the left lung might have been due to the filling up of the left pleural cavity with abdominal viscera. Indeed, the left lung might have been of normal size at the time of herniation of the abdominal viscera and then it might have filled the pleural space.

Herniation of the MiD-gut LoOP.-The viscera which are the first to enter the pleural cavity are those which return from the umbilical cord to the abdominal cavity in the tenth week of $\underset{\vec{\rho}}{\stackrel{\vec{\rho}}{*}}$ foetal life. The sudden return of these viscera into the abdominal cavity must raise the intra- 흠 abdominal pressure, and, if there is a hiatus in the diaphragm, this pressure will be relieved by the same viscera herniating into the left pleural cavity. The mid-gut loop entering the pleural cavity will present its pre-axial coils before its post-axial coils, and the ascending colon, which shares the common mid-gut mesentery, will follow the postaxial coils of the small intestine, accounting for the sequence of herniation of the small and large intestine in the hernias of Bochdalek. The stomach with its fundus opposite the hiatus in the diaphragm might have been expected to herniate first, but the mid-gut loop entering the abdominal cavity from the umbilical cord has a greater mobility and lesser bulk, giving it priority of entry over the stomach.

Herniation of the Stomach.-The liver, now unrestrained in its growth by the herniation of the mid-gut loop, will fill the upper abdomen more completely and this may set up the additional pressures which determine the herniation of the transverse colon and the stomach. When the stomach herniates it will enter the pleural cavitक fundus first, and, in doing so, the fundus wit rotate around the medial margin of the hiatus in the diaphragm, because it is anchored to the oesophagus at this place. A rotation through $180^{\circ}$ in the coronal plane carries the fundus of the stomach against the mediastinum, and when this rotation is arrested the whole of the stomach has been drawn into the pleural cavity where it lies with its greater curve uppermost and with its lesser curve just above the diaphragm.

Visceral Displacements After Herniation of MID-GUT LoOP.- The C curve of the duodenum is anchored to the hilum of the liver and is therefore retained in the abdomen, but the herniation of the stomach into the pleural cavity has pulled on the proximal limb of the $\mathrm{C}$ curve till the duodenum passes vertically down from the pylorus. The distal limb of the $C$ curve of the duodenum continues as the limb of the small intestine, ascending vertically into the pleural cavity behind the $N$ stomach. Consequently the duodenum now lies $\mathrm{N}$ with its concavity directed upwards. The head of the pancreas is fixed in the $C$ curve of the duodenum, and the body of the pancreas, attached by its tail to the spleen at the fundus of the stomach, is swung from the horizontal to the vertical position.

In the two infants dissected, the transverse colon and the splenic flexure of the colon were attached 
to the greater curve of the stomach as was also the spleen. These viscera must have been so attached before the stomach herniated into the pleural cavity. In the embryo, the dorsal mesogastrium (greater omentum) is fused with the mesentery of the transverse colon long before the reduction of the mid-gut loop from the umbilical cord into the abdominal cavity (Kanagasuntheram, 1957). This early fusion of the left colon to the stomach determines their entry together into the pleural cavity.

\section{The Origin of the Hiatus of Bochdalek}

Keith (1910) investigated 34 specimens of congenital diaphragmatic hernia and found that 17 had a defect in the dorsal or spinal part of the diaphragm on the left side and four had a defect at a corresponding position on the right. Keith termed these defects or openings in the diaphragm "the pleuro-peritoneal triangles," which he considered to be triangular intervals between the vertebral and costal elements of the diaphragm. Brash (1952) describes the vertebro-costal triangle as an

"important triangular gap found in many bodies between the vertebral and costal attachments, above the lateral part of the last rib behind the kidney. It is usually larger on the left side, and more often present on that side. It is the remains of an interval that existed between parts of the diaphragm of different developmental origin, and through which the pleural and peritoneal cavities were originally continuous. Its practical importance is that on the left side some of the contents of the abdomen may be herniated into the thorax."

A similar view with regard to the vertebro-costal triangle is held by Gruber (1927).

Harrington (1945) illustrates the hiatus within the muscular part of the diaphragm, but makes no comment in his text. Wells (1954) asserts that the hiatus is in the costal part of the diaphragm, and not between its costal and vertebral attachments. The dissections of the two infants reported in this paper establish that the hiatus of Bochdalek is ventral to the vertebro-costal triangles which were clearly present in these two cases.

It is agreed that the hiatus in the diaphragm in the hernias of Bochdalek are from a persistence of the pleuro-peritoneal openings. The anlage of the diaphragm is the septum transversum, dorsal to which are the pleuro-peritoneal canals which are subsequently walled off by extensions of the diaphragm, but in the hernias of Bochdalek this process is not achieved.

In the normal case, the closure at the site of the pleuro-peritoneal openings could have been either from an extension of the septum transversum to the body wall, or from a concentric closure of the openings from tissues surrounding these openings. In the female infant reported in this paper, the hiatus was in the muscular (costal) part of the diaphragm, and this gives proof of a concentric closure of the pleuro-peritoneal opening. In the male infant, the hiatus reached out to the body wall, and, in this case, the extension from the body wall must have been suppressed. The demonstration of a separate vertebro-costal triangle in this case too shows that the hiatus of Bochdalek could be present independently of this triangle, even when the hiatus reached to the body wall.

The vertebro-costal triangles of the diaphragm are behind the upper poles of the kidneys in the adult. In the human embryo, however, the mesonephros would have extended through the vertebro-costal triangles, and it is possible that, apart from marking the interval between the vertebral and costal attachments of the diaphragm, these triangles also mark the sites at which the mesonephros runs dorsal to the septum transversum. The fact that the musculature of the diaphragm is well established before the disappearance of the mesonephros supports this view.

\section{SUMMARY}

The origin of the hernial orifice and the mechanics of herniation have been analysed from the anatomical details studied at necropsy in two cases of congenital left-sided diaphragmatic hernia. This analysis has permitted the formulation of definite concepts in regard to the site of the foramen of Bochdalek and to the process of herniation which occurs when the foramen is patent. Surgical reduction of the hernial contents into the abdominal cavity will be more readily performed when the manipulation reverses the path taken by the viscera during the process of herniation.

We should like to thank Mr. Frank B. Price for the care and skill with which he prepared the illustrations for this article.

\section{REFERENCES}

Bochdalek (1848). Vjschr. prakt. Heilk, 19, 89.

Brash, J. C. (1952). Cunningham's Manual of Practical Anatomy, 11 th ed., vol. 2, p. 362 . Oxford University Press, London.

Dickson, W. H. (1933). Canad. med. Ass. J., 29, 24.

Gross, R. E. (1953). The Surgery of Infancy and Childhood. Saunders, Philadelphia.

Gruber, G. B. (1927). In Schwalbe, E., Die Morphologie der Missbildungen des Menschen und der Tiere, teil 3, xii, lieferung, p. 94. Fischer, Jena.

Harrington, S. W. (1945). Ann. Surg., 122, 546.

Kanagasuntheram, R. (1957). J. Anat. (Lond.), 91, 188.

Keith, A. (1910). Brit. med. J., 2, 1297.

Rickham, P. P. (1955). Thorax, 10, 104.

Wells, L. J. (1954). Contr. Embryol. Carneg. Instn, 35, 135. 